\title{
Necessary optimality conditions in nonsmooth semi-infinite multiobjective optimization under metric subregularity
}

\author{
Nguyen Dinh Huy ${ }^{1}$, Cao Thanh Tinh ${ }^{2}$, Nguyen Minh Tung ${ }^{3, *}$, Cao Thi Be Oanh ${ }^{4}$
}

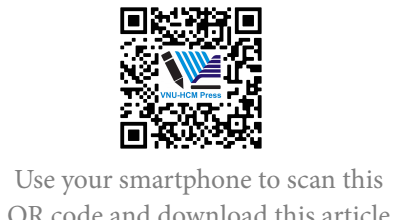

QR code and download this article

${ }^{1}$ University of Technology, VNU-HCM, Vietnam

${ }^{2}$ University of Information Technology, VNU-HCM, Vietnam

${ }^{3}$ University of Science, VNU-HCM, Vietnam

${ }^{4}$ Can Tho University of Technology, Vietnam

Correspondence

Nguyen Minh Tung, University of Science, VNU-HCM, Vietnam

Email: nmtung@hcmus.edu.vn

History

- Received: 04-12-2019

- Accepted: 28-12-2020

- Published: 31-12-2020

DOI : 10.32508/stdjet.v3iSI3.637

\section{Check for updates}

Copyright

(c) VNU-HCM Press. This is an openaccess article distributed under the terms of the Creative Commons Attribution 4.0 International license.

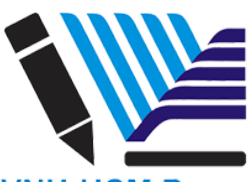

VNU-HCM Press

ABSTRACT

We consider nonsmooth semi-infinite multiobjective optimization problems under mixed constraints, including infinitely many mixed constraints by using Clarke subdifferential. Semi-infinite programming (SIP) is the minimization of many scalar objective functions subject to a possibly infinite system of inequality or/and equality constraints. SIPs have been proved to be very important in optimization and applications. Semi-infinite programming problems arise in various fields of engineering such as control systems design, decision-making under competition, and multi-objective optimization. There is extensive literature on standard semi-infinite programming problems. The investigation of optimality conditions for these problems is always one of the most attractive topics and has been studied extensively in the literature. In our work, we study optimality conditions for weak efficiency of a multi-objective semi-infinite optimization problem under mixed constraints including infinitely many of both equality and inequality constraints in terms of Clarke sub-differential. Our conditions are the form of the Karush-Kuhn-Tucker (KKT) multiplier. To the best of our knowledge, only a few papers are dealing with optimality conditions for SIPs subject to mixed constraints. By the Pshenichnyi-Levin-Valadire (PLV) property and the directional metric sub-regularity, we introduce a type of Mangasarian-Fromovitz constraint qualification (MFCQ). Then we show that (MFCQ) is a sufficient condition to guarantee the extended Abadie constraint qualification (ACQ) to satisfy. In our constraint qualifications, all functions are nonsmooth and the number of constraints is not necessarily finite. In our paper, we do not need the involved functions: convexity and differentiability. Later, we apply the extended Abadie constraint qualification to get the KKT multipliers for weak efficient solutions of SIP. Many examples are provided to illustrate some advantages of our results. The paper is organized as follows. In Section Preliminaries, we present our basic definitions of nonsmooth and convex analysis. Section Main Results prove necessary conditions for the weakly efficient solution in terms of the Karush-Kuhn-Tucker multiplier rule with the help of some constraint qualifications.

Key words: Optimality condition, SIP, constraint qualification, weak efficiency, metric subregularity

\section{INTRODUCTION}

Semi-infinite optimization (SIP) is the simultaneous minimization of finitely many scalar objective functions under an arbitrary set of inequality constraints or/and equality constraints. (SIPs) arise in many fields of applied mathematics such as robotics, control system design, etc, see for instance ${ }^{1-3}$. Investigation of optimality conditions for SIPs has been considered extensively in the literature.

With linear semi-infinite systems, Goberna ${ }^{4}$ introduced he Farkas-Minkowski property, Puenten and Vera ${ }^{5}$ proposed the local Farkas-Minkowski property and used it as a constraint qualification to get Lagrange multipliers. For convexsemi-infinite optimization, many constraint qualifications have been studied in Lopez and Vercher ${ }^{6}$. With the help of the Abadie constraint qualification, optimality conditions for semi-infinite systems of convex and linear inequalities were developed in $\mathrm{Li}^{7}$. For smooth problems, Stein ${ }^{8}$ proposed the Abadie and MangasarianFromovitz constraint qualifications to conisder optimality conditions. By employing variational analysis, Mordukhovich and Nghia ${ }^{9}$ obtained necessary conditions under the extended perturbed MangasarianFromovitz and Farkas--Minkowski constraint qualification. For nonsmooth problem with inequality constraints, Zheng and Yang ${ }^{10}$ employed the directional derivative to obtain Lagrange multiplier rules. Kanzi and Nobakhtian ${ }^{11,12}$ introduced several nonsmooth analogues of the Abadie constraint qualification and the Pshenichnyi-Levin-Valadire property and applied them to obtain optimality conditions. Chuong ${ }^{13}$ proposed the limiting constraint qualification in terms of the Mordukhovich subdifferential and applied it to optimality conditions. Kanzi ${ }^{14}$ investigated nonsmooth semi-infinite problems with mixed constraints by the Michel-Penot subdifferential. We observe that Engineering and Technology; 3(SI3):SI52-SI57. 
the constraints in the above mentioned papers contain finitely many equalities. There are very few publications dealing with infinitely many equality constraints.

In this paper we investigate opyimality conditions for weak efficiency of a multiobjective semi-infinite optimization problem under mixed constraints including infinitely many of both equality and inequality constraints in terms of Clarke subdifferential. By the Pshenichnyi-Levin-Valadire (PLV) property and the directional metric subregularity, we propose Mangasarian-Fromovitz constraint qualification (MFCQ) to guarantee the extend Abadie constraint qualification (ACQ) to satisfy. In our constraint qualifications, all functions are nonsmooth and the number of the equality constraints is not necessary finite. Then, we apply them to get the KKT multipliers. The paper is organized as follows. In Section Prelininaries, we present our basic definitions. Section Main results prove necessary conditions for weak efficiency in terms of Karush-Kuhn-Tucker multiplier under some constraint qualification.

\section{RELIMINARIES}

$\mathbb{N}, \mathbb{R}^{n}$ and $\mathbb{R}_{+}^{n}$ stand for the set of the natural numbers, a n-dimensional vector space and its nonnegative orthant, respectively (resp). $B(x, r)$ denotes the open ball with centre $x$ and radius $r$. For $M \subset \mathbb{R}^{n}$, int $M, c l M, b d M$ and $c o M$ stand for its interior, closure, boundary, and convex hull of $M$, resp. The cone hull of $M$ is defined by cone $M:=$ $\{\lambda x ? ? ?, x \in M\}$. The contingent cone of $M \subset$ $\mathbb{R}^{n}$ at $\bar{x} \in \mathrm{cl} M$ is

$$
\begin{gathered}
T(M, \bar{x}):=\left\{d \in \mathbb{R}^{n}, t_{n} 0 \rightarrow 0^{+}, \exists d_{n} \rightarrow d,\right. \\
\left.\bar{x}+t_{n} d_{n} \in M, \forall n \in \mathbb{N}\right\} .
\end{gathered}
$$

A map $f: \mathbb{R}^{n} \rightarrow \mathbb{R}$ is locally Lipschitz at $x_{0} \in \mathbb{R}^{n}$ if there is a neighborhood $U$ of $x_{0}$ and a real number $x_{0} \in \mathbb{R}^{n}$ such that

$\|f(x)-f(y)\| \leq L\|x-y\|, \forall x, y \in U$.

A set-valued map $F: \mathbb{R}^{n} \rightarrow 2^{\mathbb{R}^{m}}$ is concave if $\forall a, b \in \mathbb{R}^{n}, \forall \lambda \in[0,1]$,

$\lambda F(a)+(1-\lambda) F(b) \subseteq F(\lambda a+(1-\lambda) b)$.

Definition 2.1. $\left({ }^{15}\right)$

Let $f: \mathbb{R}^{n} \rightarrow \mathbb{R}$ and $x_{0}, d \in \mathbb{R}^{n}$. The Clarke directional derivative of $f$ at $x_{0}$ in direction $d$ is

$f^{0}\left(x_{0}, d\right):=\limsup _{x \rightarrow x_{0}, t \rightarrow 0^{+}} \frac{f(x+t d)-f(x)}{t}$ and the Clarke subdifferential of $f$ at $x_{0}$ is

$\partial_{C} f\left(x_{0}\right):=$

$\left\{x^{*} \in \mathbb{R}^{n} ? ? f^{0}\left(x_{0}, d\right) \geq\left\langle x^{*}, d\right\rangle, \forall d \in \mathbb{R}^{n}\right\}$.

Recall that the directional of $f$ at $x_{0}$ in direction $d$ is $f^{\prime}\left(x_{0}, d\right):=\limsup _{t \rightarrow 0^{+}} \frac{f\left(x_{0}+t d\right)-f\left(x_{0}\right)}{t}$

$f$ is regular at $x_{0}$ if $f^{0}\left(x_{0}, d\right)=f^{\prime}\left(x_{0}, d\right)$.
The following properties will be useful in the sequel $\left({ }^{15}\right)$.

\section{Proposition 2.1}

Let $f, g: \mathbb{R}^{n} \rightarrow \mathbb{R}$ be locally Lipschitz at $x_{0} \in \mathbb{R}^{n}$ and $d \in \mathbb{R}^{n}$.

(i) $d \mapsto f^{0}\left(x_{0}, d\right)$ is finite, positivel homogeneous and subadditive on $\mathbb{R}^{n}$, and $\partial\left(f^{0}\left(x_{0}, \cdot\right)(0)=\partial_{C} f\left(x_{0}\right)\right.$, where $\partial$ denotes the subdifferential in the sense of convex analysis.

(ii) $\partial_{C} f\left(x_{0}\right)$ is a nonempty, convex and compact subset of $\mathbb{R}^{n}$ and, for every

$d \in \mathbb{R}^{n}, f^{0}\left(x_{0}, d\right)=\max _{x^{*} \in \partial_{C} f\left(x_{0}\right)}\left\langle x^{*}, d\right\rangle$.

(iii) $\partial_{C}(f+g)\left(x_{0}\right) \subseteq \partial_{C} f\left(x_{0}\right)+\partial_{C} g\left(x_{0}\right)$. If in addition both $f$ and $g$ are regular at $x_{0}$, then the equality holds.

(iv) If $x_{0}$ is a local minimum of $f$, then $0 \in \partial_{C} f\left(x_{0}\right)$.

Besides single-valued directional derivatives, we need the following set-valued directional derivatives.

\section{Definition 2.2}

The Hadamard set-valued directional derivative of $f$ : $\mathbb{R}^{n} \rightarrow \mathbb{R}$ at $x_{0} \in \mathbb{R}^{n}$ in direction $d_{0} \in \mathbb{R}^{n}$ is

$$
\begin{aligned}
& D f\left(x_{0}, d\right):=\left\{y \in \mathbb{R}^{m} ? ? t_{n} \rightarrow 0^{+}, \exists d_{n} \rightarrow d,\right. \\
& \left.y=\lim _{n \rightarrow \infty} t_{n}^{-1}\left(f\left(x_{0}+t_{n} d_{n}\right)-f\left(x_{0}\right)\right)\right\}
\end{aligned}
$$

Definition $2.3\left({ }^{16}\right)$

$f: \mathbb{R}^{n} \rightarrow \mathbb{R}, x_{0} \in \mathbb{R}^{n}$, and $y_{0}=f\left(x_{0}\right) . f$ is said to bedirectionally metrically subregular at $x_{0}$ in direction $d$ if there are a neighborhood $U$ of $x_{0}, a \geq 0$, and $r>0$, for $t \in(0, r)$ and $v \in B_{X}(d, r), d\left(x_{0}+t v, f^{-1}\left(y_{0}\right)\right) \leq$ $\operatorname{ad}\left(y_{0}, f\left(x_{0}+t v\right)\right)$.

\section{Proposition 2.2}

$f: \mathbb{R}^{n} \rightarrow \mathbb{R}, x_{0} \in \mathbb{R}^{n}$, and $y_{0}=f\left(x_{0}\right)$. If $0 \notin$ $D f\left(x_{0}\right)(d)$ then $f$ is directionally metrically subregular at $x_{0}$ in direction $d$.

Proof. Suppose there are $t_{n} \rightarrow 0$ and $d_{n} \rightarrow d$ such that, for all $n$,

$d\left(x_{0}+t_{n} d_{n}, f^{-1}\left(y_{0}\right)\right)>n d\left(y_{0}, f\left(x_{0}+t_{n} d_{n}\right)\right)$. Then, there exists $y_{n}=f\left(x_{0}+t_{n} d_{n}\right)$ such that

$\left\|y_{n}-y_{0}\right\|<n^{-1}\left\|\left(x_{0}+t_{n} d_{n}\right)-x_{0}\right\|$,

$t_{n}^{-1}|| y_{n}-y_{0}\left\|<n^{-1}\right\| d_{n} \|$.

By setting $v_{n}=t_{n}^{-1}\left(y_{n}-y_{0}\right)$, one has $v_{n} \rightarrow 0$ and $y_{0}+t_{n} v_{n}=f\left(x_{0}+t_{n} d_{n}\right)$, i.e., $0 \in D f\left(x_{0}\right)(d)$, which contradicts the assumption.

The following example present that the sufficient condition given in Proposition 2.2 is not necessary.

Example 2.1 Let $f: \mathbb{R}^{2} \rightarrow \mathbb{R}$ be defined by $f\left(x_{1}, x_{2}\right)=\left|x_{1}-x_{2}\right|$ and $d_{1}=(1,0)$. We can check that $0 \notin D f(0)\left(d_{1}\right)=\{1\}$, hence the assumption of Proposition 2.2 is fulfilled. By calculations, we have that

$d\left(t v, f^{-1}(0)\right)=2^{-1 / 2} t\left|v_{1}-v_{2}\right|$, $d(0, f(t v))=t\left|v_{1}-v_{2}\right|$ 
for $v=\left(v_{1}, v_{2}\right) \in \mathbb{R}^{2}$ and $t \in \mathbb{R}_{+}$with $t v \notin f^{-1}(0)$. Then, for $r>0, t \in(0, r)$, and $v \in B\left(d_{1}, r\right)$, $\frac{t}{\sqrt{2}}\left|v_{1}-v_{2}\right| \leq t\left|v_{1}-v_{2}\right|$,

i.e., $d\left(t v, f^{-1}(0)\right) \leq d(0, f(t v))$.

Hence, $f$ is directionally metrically subregular at 0 in direction $d_{1}$ in Proposition 2.2. Now we replace $d_{1}$ by $d_{2}=(1,1)$. Similarly, we check that the above inequality holds for $r>0, t \in(0, r)$, and $v \in B\left(d_{2}, r\right)$. However, $0 \in D f(0,0)\left(d_{2}\right)=\{0\}$..

\section{MAIN RESULTS}

We investigate the fmultiobjective semi-infinite optimization problem under mixed constraints:

(P) $\min ^{\mathbb{R}_{+}^{\mathrm{m}}}$ s.t. $\left\{\begin{array}{l}g_{i}(x) \leq 0, i \in I \\ h_{j}(x) \leq 0, j \in J\end{array}\right.$

where $f:=\left(f_{1}, \ldots, f_{m}\right): \mathbb{R}^{n} \rightarrow \mathbb{R}^{m}, g_{i}: \mathbb{R}^{n} \rightarrow \mathbb{R}$ for $i \in I$, and $h_{j}: \mathbb{R}^{n} \rightarrow \mathbb{R}$ for $j \in J$, are locally Lipschitz. The index sets $I$ and $J$ are arbitrary. The feasible set of problem $(\mathrm{P})$ is

$$
\Omega:=\left\{x \in \mathbb{R}^{n} ?\left\{\begin{array}{c}
g_{i}(x) \leq 0, i \in I \\
h_{j}(x) \leq 0, j \in J
\end{array}\right\} .\right.
$$

Definition 3.1 For the problem (P) and $x_{0} \in \Omega$. $x_{0}$ is called a local weak efficient solution of $(\mathrm{P})$, written as $x_{0} \in L W(P)$, if there is a neighborhood $U$ of $x_{0}$ such that

$(f(U \cap \Omega)-f(x)) \cap\left(-\operatorname{int} \mathbb{R}_{+}^{m}\right)=\varnothing$.

We denote $I\left(x_{0}\right):=\left\{i \in I ? g_{i}\left(x_{0}\right)=0\right\}$ and

$$
\begin{aligned}
& \left\{d\left(\Omega, x_{0}\right):=\right. \\
& \left\{d \in X ?\left\{\begin{array}{l}
g_{i}^{0}\left(x_{0}, d\right) \leq 0, \forall i \in I\left(x_{0}\right) \\
h_{i}^{0}\left(x_{0}, d\right)=0, \forall j \in J\left(x_{0}\right)
\end{array}\right\}\right\}, \\
& \triangle:=\bigcup_{i \in I\left(x_{0}\right)} \partial_{C} g_{i}\left(x_{0}\right) \cup \\
& \bigcup_{j \in J}\left(\partial_{C} h_{j}\left(x_{0}\right) \cup \partial_{C}\left(-h_{j}\right)\left(x_{0}\right)\right),
\end{aligned}
$$

$G(x) \quad:=\quad \sup _{i \in I} g_{i}(x), \quad H(x) \quad:=$

$\sup _{j \in J} \max \left\{h_{j}(x),-h_{j}(x)\right\}$.

Definition 3.2 $\left({ }^{5}\right)$ The Pshenichnyi-Levin-Valadire (PLV) property holds at $x_{0} \in \Omega$ with respect to (wrt) $G$ iff $G$ is locally Lipschitz around $x_{0}$ and $\partial_{C} G\left(x_{0}\right) \subset$ $\operatorname{conv} \bigcup_{i \in I\left(x_{0}\right)} \partial_{C} g_{i}(x)$.

If I is finite and $g_{i}$ are locally Lipschitz around $x_{0}$ for $i \in I$, obviously the problem (P) has the PshenichnyiLevin-Valadire (PLV) property at $x_{0} \in \Omega$ wrt G. Sufficient conditions for $G$ to be locally Lipschitz were considered $^{17}$.

Definition 3.3 For (P) and $x_{0} \in \Omega$.

(i) The extended Abadie constraint qualification (ACQ) satisfies at $x_{0}$ if $L\left(\Omega, x_{0}\right)=T\left(\Omega, x_{0}\right)$.

(ii) The extend Mangasarian-Fromovitz constraint qualification (MFCQ) satisfies at $x_{0}$ if there exists $\bar{d}$ such that (a) $g_{i}^{0}\left(x_{0}, \bar{d}\right)<0$ for all $i \in I\left(x_{0}\right)$;

(b) $H$ is directionally metrically subregular at $x_{0}$, $D H\left(x_{0}, \cdot\right)$ is concave on $X, h_{j}$ is regular for all $j \in J$, and $0 \in D H\left(x_{0}, \bar{d}\right)$.

Theorem 3.1 If $(P)$ has the (PLV) property at $x_{0} \in \Omega$ wrt $G$ and the (MFCQ) satisfies at $x_{0}$, then the (ACQ) satisfies at $x_{0}$.

Proof. By the (MFCQ), there is $\bar{d}$ such that $g_{i}^{o}\left(x_{0}, \bar{d}\right)<0$ for all $i \in I\left(x_{0}\right)$. This implies that

$\left\langle x^{*}, \bar{d}\right\rangle<0, \forall x^{*} \in \bigcup_{i \in I\left(x_{0}\right)} \partial_{C} g_{i}(x)$,

$\left\langle x^{*}, \bar{d}\right\rangle<0, \forall x^{*} \in \operatorname{conv}\left(\bigcup_{i \in I\left(x_{0}\right)} \partial_{C} g_{i}(x)\right)$.

By (PLV), one has $\left\langle x^{*}, \bar{d}\right\rangle<0$ for all $x^{*} \in \partial_{C} G\left(x_{0}\right)$ and so $G_{0}\left(x_{0}, \bar{d}\right)<0$. Then, $\limsup _{t \rightarrow 0^{+}} \frac{G^{0}\left(x_{0}+t \bar{d}\right)-G\left(x_{0}\right)}{t} \leq G^{0}\left(x_{0}, \bar{d}\right)<0$, which implies there are $\beta$ and $\varepsilon$ such that

(1) $G\left(x_{0}+t \bar{d}\right)-G\left(x_{0}\right)<-t \beta, \forall t \in(0, \varepsilon)$. Besides, as $0 \in D H\left(x_{0}, \bar{d}\right)$, there exist $t_{n} \rightarrow 0^{+}, d_{n} \rightarrow$ $\bar{d}$, and $z_{n} \rightarrow 0$ such that $t_{n} z_{n} \in H\left(x_{0}+t_{n} d_{n}\right)$. The metric subregularity of $H$ gives $a \geq 0$ such that, for large $n$,

$$
\begin{gathered}
d\left(x_{0}+t_{n} d_{n}, H^{-1}(0)\right) \leq a d\left(0, H\left(x_{0}+t_{n} u_{n}\right)\right) \\
\leq a t_{n}\left\|z_{n}\right\| .
\end{gathered}
$$

Hence, there exist $\bar{d}_{n}$ and $\varepsilon$ with $t_{n}^{-1} \varepsilon_{n} \rightarrow 0^{+}$such that $x_{0}+t_{n} \bar{d} \in H^{-1}(0)$ and $\left\|\left(x_{0}+t_{n} u_{n}\right)-\left(x_{0}+t_{n} \bar{u}_{n}\right)\right\| \leq$ $a t_{n}\left\|z_{n}\right\|+\varepsilon_{n}$.

Then, $\bar{d}_{n} \rightarrow d$. Since $x_{0}+t_{n} \bar{d}_{n} \in H^{-1}(0)$, one has, $\max \left\{h_{j}\left(x_{0}+t_{n} \bar{d}_{n}\right),-h_{j}\left(x_{0}+t_{n} \bar{d}_{n}\right)\right\} \leq 0$,

Hence, for large $n$,

(2) $h_{j}\left(x_{0}+t_{n} \bar{d}_{n}\right)=0, \forall j \in J$.

From (1), one has, for large $n$,

$G\left(x_{0}+t_{n} \bar{d}_{n}\right)-G\left(x_{0}\right)<-t_{n} \beta$.

Since $G$ is locally Lipschitz at $x_{0}$, there is $L>0$ such that, for large $n$,

$$
\begin{aligned}
& G\left(x_{0}+t \bar{d}_{n}\right)-G\left(x_{0}+t_{n} \bar{d}_{n}\right) \leq L t_{n}\left\|\bar{d}_{n}-\bar{d}\right\|, \\
& G\left(x_{0}+t \bar{d}_{n}\right) \leq G\left(x_{0}+t \bar{d}_{n}\right)+L t_{n}\left\|\bar{d}_{n}-\bar{d}\right\| \\
& <G\left(x_{0}\right)+t_{n}\left(-\beta+L\left\|\bar{d}_{n}-\bar{d}\right\|\right) \leq 0 .
\end{aligned}
$$

This implies that $g_{i}\left(x_{0}+t \bar{d}_{n}\right) \leq 0$ for all $i \in I$. By combining this and (2), one has $x_{0}+t \bar{d}_{n} \in \Omega$. Hence, $\bar{d} \in T\left(\Omega, x_{0}\right)$.

Let $d \in L\left(\Omega, x_{0}\right)$, we prove $d \in T\left(\Omega, x_{0}\right)$.

Set $d_{n}=n^{-1} \bar{d}+\left(1-n^{-1}\right) d$ for $n \geq 2$.

By Proposition 2.1, for all $i \in I\left(x_{0}\right)$ one has

(3) $g_{i}^{0}\left(x_{0}, d_{n}\right) \leq n^{-1} g_{i}^{0}\left(x_{0}, \bar{d}_{n}\right)+$ $\left(1-n^{-1}\right) g_{i}^{0}\left(x_{0}, \bar{d}_{n}\right)<0$. Since $h_{j}$ is regular at $x_{0}$ and $d \in L\left(\Omega, x_{0}\right)$, one gets for all $j \in J$, $h_{j}^{\prime}\left(x_{0}, d\right)=h_{j}^{0}\left(x_{0}, d\right)=0$ and

$\lim _{t \rightarrow 0^{+}} \frac{h_{j}\left(x_{0}+t d\right)-h_{j}\left(x_{0}\right)}{t}=\lim _{t \rightarrow 0^{+}} \frac{h_{j}\left(x_{0}+t d\right)}{t}=0$. 
Then, there exists $t_{n} \rightarrow 0^{+}$such that $\lim _{\mathrm{n} \rightarrow \infty} \frac{\max \left\{h_{j}\left(x_{0}+t_{n} d\right),-h_{j}\left(x_{0}+t_{n} d\right)\right\}}{t_{n}}=0$. Hence, $0 \in D H\left(x_{0}, d\right)$.

Because $D H\left(x_{0}, \cdot\right)$ is concave,

$$
\begin{aligned}
& n^{-1} D H\left(x_{0}, \bar{d}\right)+\left(1-n^{-1}\right) D H\left(x_{0}, d\right) \\
& \in D H\left(x_{0}, n^{-1} \bar{d}+\left(1-n^{-1}\right) d\right) .
\end{aligned}
$$

Hence,

(4) $0 \in \mathrm{DH}\left(x_{0}, d_{n}\right)$

From (3) and (4), similar to the above arguments, one has $d_{n} \in T\left(\Omega, x_{0}\right)$. As $d_{n} \rightarrow d$ and is a closed cone, $d \in T\left(\Omega, x_{0}\right)$.

The proof is complete.

Remark 3.1

Nonsmooth SIPs involving mixed constraints ${ }^{9,14}$, the (MFCQ) was used to consider a number of equality constraints. In these paper, the functions were continuously differentiable with the linearly independent gradients such that $\left\langle\nabla f_{j}\left(x_{0}\right), \bar{d}\right\rangle=0$ for $j \in J$. The inequality constraints were continuously differentiable and the equalities werestrictly differentiable. By employing directional metric subregularity, out (MFCQ) can be used to nonsmooth infinite mixed constraint systems and the condition $0 \in D H\left(x_{0}, \bar{d}\right)$ can be applied in many cases..

The next example provides a case where Theorem 3.1 can be employed, while many MangasarianFromovitz-type constraint qualifications cannot.

Example 3.1 Let $g_{i}, h_{j}: \mathbb{R}^{2} \rightarrow \mathbb{R}$ be defined by for $i \in$ $N$

$g_{1}\left(x_{1}, x_{2}\right)=x_{1}, g_{2 i+1}\left(x_{1}, x_{2}\right)=x_{1}-i^{-1}$,

$g_{2}\left(x_{1}, x_{2}\right)=x_{2}, g_{2 i+2}\left(x_{1}, x_{2}\right)=$

$x_{2}-(1+i)^{-1}, h_{j}\left(x_{1}, x_{2}\right)=j\left(x_{1}-x_{2}\right), j \in(0,1)$.

Hence, $\Omega=\left\{\left(x_{1}, x_{2}\right) \in \mathbb{R}^{2} \mid x_{1}=x_{2} \leq 0\right\}$.

For $x_{0}=(0,0), I\left(x_{0}\right)=\{1,2\}$. We see that

$G\left(x_{1}, x_{2}\right)=\sup \left\{x_{1}, x_{2}\right\}, H\left(x_{1}, x_{2}\right)=\left|x_{1}-x_{2}\right|$ are locally Lipschitz at $x_{0}$ and $\partial_{C} G\left(x_{0}\right) \subseteq$ $\operatorname{conv} \bigcup_{i \in I\left(x_{0}\right)} \partial_{C} g_{i}(x)$. Thus, (P) has the (PLV) property at $x_{0}$ wrt $G$. Now, we check that the (MFCQ) is fulfilled at $x_{0}$ with $\bar{d}=(1,-1)$. For $i \in I\left(x_{0}\right), j \in J, g_{i}^{0}\left(x_{0}, \bar{d}\right)=-1<0, h_{j}$ is regular, $H\left(x_{1}, x_{2}\right)=\left|x_{1}-x_{2}\right|$ and so $H$ is directionally metrically subregular at $x_{0}$ (by Example 2.1),

$D H\left(x_{0},\left(d_{1}, d_{2}\right)\right)=\operatorname{conv}\left\{\left(d_{1},-d_{2}\right) ;\left(-d_{1}, d_{2}\right)\right\}$ for all $\left(d_{1}, d_{2}\right) \in X$ and so $D H\left(x_{0}, \cdot\right)$ is concave, and $0 \in D H\left(x_{0}, \bar{d}\right)$. Therefore, the (MFCQ) holds at $x_{0}$. By Theorem 3.1, the (ACQ) holds at $x_{0}$. (We can check the (ACQ) by direct calculations as follows. As $g_{1}^{0}\left(x_{0}, d\right)=d_{1}, g_{2}^{0}\left(x_{0}, d\right)=d_{2}$, and $h_{j}^{0}\left(x_{0}, d\right)=$ $j\left(d_{1}-d_{2}\right)$, we have

$L\left(\Omega, x_{0}\right)=T\left(\Omega, x_{0}\right)=\left\{\left(d_{1}, d_{2}\right) \in \mathbb{R}^{2} ? d_{1}=d_{2} \leq 0\right\}$ and so (ACQ) holds. Because $\mathrm{J}$ infinite, the (MFCQ) $)^{9,14}$ cannot be employed.
The following example shows the essentialness of the directional metric subregularity of $\mathrm{H}$.

Example 3.2 Let $g_{i}$ be the same as in Example 3.1 and $h_{j}\left(x_{1}, x_{2}\right)=j\left(x_{1}^{2}-x_{2}^{2}\right), j \in(0,1)$.

Hence, $\quad \Omega=\left\{\left(x_{1}, x_{2}\right) \in \mathbb{R}^{2} ? ? \tau_{1}=\tau_{2} \leq 0\right\} \quad$ and $I\left(x_{0}\right)=\{1,2\}$ for $x_{0}=(0,0)$. Similar to Example $3.1,(\mathrm{P})$ has the (PLV) property at $x_{0}$ wrt $G$. We check that the (MFCQ) holds at $x_{0}$ for $\bar{d}=(1,-1)$. We have $g_{i}^{0}\left(x_{0}, \bar{d}\right)=-1<0$ for all $i \in I\left(x_{0}\right)$.

$h_{j}$ is regular, $j \in J, H\left(x_{1}, x_{2}\right)=$ $\left|x_{1}^{2}-x_{2}^{2}\right|, D H\left(x_{0},\left(d_{1}, d_{2}\right)\right)=\{0\}$ for $\left(d_{1}, d_{2}\right) \in X$ and so $D H\left(x_{0}, \cdot\right)$ is concave, and $0 \in D H\left(x_{0}, \bar{d}\right)$. On the other hand, as $g_{1}^{0}\left(x_{0}, d\right)=0, g_{2}^{0}\left(x_{0}, d\right)=d_{2}$, and $h_{j}^{0}\left(x_{0}, d\right)=0$, we have

$L\left(\Omega, x_{0}\right)=\left\{\left(d_{1}, d_{2}\right) \in \mathbb{R}^{2} ? d_{1} \leq 0, d_{2} \leq 0\right\}$,

$T\left(\Omega, x_{0}\right)=\left\{\left(d_{1}, d_{2}\right) \in \mathbb{R}^{2} ? d_{1}=0, d_{2}=0\right\}$,

$L\left(\Omega, x_{0}\right) \neq T\left(\Omega, x_{0}\right)$.

The cause is that $H$ is not directionally metrically subregular at $x_{0}$. We have

$d\left(t v, H^{-1}(0)\right)=1 / \sqrt{2} \min \left\{\left|v_{1}+v_{2}\right|,\left|v_{1}-v_{2}\right|\right\}$

and $d(0, H(t v))=t^{2}\left|v_{1}^{2}-v_{2}^{2}\right|$ for $v=\left(v_{1}, v_{2}\right) \in \mathbb{R}^{2}$ and $t \in \mathbb{R}_{+}$with $t v \notin H^{-1}(0)$. Then, the subregularity means that for any $a, r>0, t \in(0, r)$, and $v \in B_{x}(\bar{d}, r)$,

$\frac{t}{\sqrt{2}} \min \left\{\left|v_{1}+v_{2}\right|,\left|v_{1}-v_{2}\right|\right\} \leq a t^{2}\left|v_{1}^{2}-v_{2}^{2}\right|$.

But, this does not hold.

Now, by employ the extend ACQ, we present a necessary optimality condition for weak efficiency of problem (P), as follows.

Theorem 3.2 Let $x_{0}$ be a local weak efficiency of $(P)$. If the (ACQ) holds at $x_{0}, \triangle$ is closed, and $f_{k}$ is regular and Lipschitz around $x_{0}$, for $k=1, \ldots . m$, then there exist $\left(\alpha_{1}, \ldots, \alpha_{m}\right) \in \mathbb{R}_{+}^{m} \backslash\{0\}, \beta_{i} \geq 0$ for $i \in I\left(x_{0}\right)$, and $\gamma_{j} \geq$ 0 for $j \in J$ such that

$0 \in \sum_{k=1}^{m} \alpha_{k} \partial_{C} f_{k}\left(x_{0}\right)+\sum_{i \in I\left(x_{0}\right)} \beta_{i} \partial_{C} g_{i}\left(x_{0}\right)$

$+\sum_{j \in J} \gamma_{j}\left(\partial_{C} h_{j}\left(x_{0}\right) \cup \partial_{C}\left(-h_{j}\right)\left(x_{0}\right)\right)$.

Proof. Step 1. We claim that the system

$\left\{f_{1}^{0}\left(x_{0}, d\right)<0, \ldots, f_{m}^{0}<0\right.$

$\left\{d \in T\left(\Omega, x_{0}\right)\right.$

has no solution. Suppose that there is $d \in T\left(\Omega, x_{0}\right)$ satisfying $f_{t}^{0}\left(x_{0}, d\right) \leq 0$ for all $t=1,2, \ldots, m$. By setting $y=\left(f_{1}^{0}\left(x_{0}, d\right), \ldots, f_{m}^{0}\left(x_{0}, d\right)\right)$, one has $y \in$ $-i n t \mathbb{R}_{+}^{m}$. As $d \in T\left(\Omega, x_{0}\right)$, there exist $t_{n} \rightarrow 0^{+}$and $d_{n} \rightarrow d$ such that $x_{0}+t_{n} d_{n} \in \Omega$ for all $n \in \mathbb{N}$. Since $f_{k}$ is regular and locally Lipschitz at $x_{0}$, one has $\lim _{n \rightarrow \infty} \frac{f_{k}\left(x_{0}+t_{n} d\right)-f_{k}\left(x_{0}\right)}{t_{n}}=f_{k}^{0}\left(x_{0}, d\right)$,

$\lim _{n \rightarrow \infty} \frac{f_{k}\left(x_{0}+t_{n} d_{n}\right)-f_{k}\left(x_{0}+t_{n} d\right)}{t_{n}}=0$,

$\lim _{n \rightarrow \infty} \frac{f_{k}\left(x_{0}+t_{n} d\right)-f_{k}\left(x_{0}\right)}{t}=$

$\lim _{n \rightarrow \infty} \frac{f_{k}\left(x_{0}+t_{n} d\right)-f_{k}\left(x_{0}\right)+f_{k}\left(x_{0}+t_{n} d_{n}\right)-f_{k}\left(x_{0}+t_{n} d\right)}{t_{n}}$.

Hence, $\lim _{n \rightarrow \infty} \frac{f\left(x_{0}+t_{n} d_{n}\right)-f\left(x_{0}\right)}{t_{n}}=y$. 
As $y \in-\operatorname{int} \mathbb{R}_{+}^{m}$, for large $n$, one has $f\left(x_{0}+t_{n} d_{n}\right)-$ $f\left(x_{0}\right) \in-\operatorname{int} \mathbb{R}_{+}^{\mathrm{m}}$, which is a contradiction. Therefore, the mentioned system has no solution.

Step 2. From Step 1, by Theorem 3.13 in $^{18}$, we have $\left(\alpha_{1}, \ldots, \alpha_{m}\right) \in \mathbb{R}_{+}^{m}$, such that

$\sum_{k=1}^{m} \alpha_{k} f_{k}^{0}\left(x_{0}, d\right) \geq 0, \forall d \in T\left(\Omega, x_{0}\right)$.

Because (ACQ) holds, one has

(5) $\sum_{k=1}^{m} \alpha_{k} f_{k}^{0}\left(x_{0}, d\right) \geq 0, \forall d \in L\left(\Omega, x_{0}\right)$.

Step 3. Denote

$\mathrm{A}=\operatorname{cone}(\operatorname{conv}(\triangle))$

and the indicator function of $\mathrm{A}^{0}$ by $\delta_{\mathrm{A}^{0}}$.

Then, (5) implies that

$\sum_{k=1}^{m} \alpha_{k} f_{k}^{0}\left(x_{0}, d\right) \geq 0, \forall d \in \mathrm{A}^{0}$.

Because $0 \in \mathrm{A}^{0}$ and $\sum_{k=1}^{m} \alpha_{k} f_{k}^{0}\left(x_{0}, 0\right)=0$,

$0 \in \operatorname{argmin}_{d \in X}\left\{\sum_{k=1}^{m} \alpha_{k} f_{k}^{0}\left(x_{0}, d\right)+\delta_{\mathrm{A}^{0}}(d)\right\}$.

By Proposition 2.1, $f_{k}^{0}\left(x_{0}, \cdot\right)$ is continuous and convex and $\mathrm{A}^{0}$ is convex. Therefore $\left({ }^{19}\right)$,

$0 \in \partial\left(\sum_{k=1}^{m} \alpha_{k} f_{k}^{0}\left(x_{0}, \cdot\right)+\delta_{A^{0}}(\cdot)\right)(0)$.

By the sum rule of subdifferentials, one has

(6) $0 \in \partial\left(\sum_{k=1}^{m} \alpha_{k} f_{k}^{0}\left(x_{0}, \cdot\right)\right)(0)+\partial \delta_{A^{0}}(\cdot)(0)$

Since $\partial f_{k}^{0}\left(x_{0}, \cdot\right)(0)=\partial_{C} f_{k}^{0}\left(x_{0}\right)$, one gets

$\partial\left(\sum_{k=1}^{m} \alpha_{k} f_{k}^{0}\left(x_{0}, \cdot\right)\right)(0)=\sum_{k=1}^{m} \alpha_{k} \partial_{k} f_{k}\left(x_{0}\right)$.

As $\triangle$ is closed, by the bipolar theorem, one has $\partial \delta_{\mathrm{A}^{0}}(0)=\left(\mathrm{A}^{0}\right)^{0}=\mathrm{A}$. Hence, from (6), there exist $\left(\alpha_{1}, \ldots, \alpha_{m}\right) \in \mathbb{R}_{+}^{m} \backslash\{0\}, \beta_{i} \geq 0$ for $i \in I\left(x_{0}\right)$, and $\gamma_{j} \geq 0$ for $j \in J$ such that

$0 \in \sum_{k=1}^{m} \alpha_{k} \partial_{k} f_{k}\left(x_{0}\right)+\sum_{i \in I\left(x_{0}\right)} \beta_{i} \partial_{C} g_{i}\left(x_{0}\right)+$

$\sum_{j \in J} \gamma_{j}\left(\partial_{C} h_{j}\left(x_{0}\right) \cup \partial_{C}\left(-h_{j}\right)\left(x_{0}\right)\right)$.

The proof is complete.

\section{Example 3.3}

Let $f: \mathbb{R}^{2} \rightarrow \mathbb{R}^{2}$ with $f=\left(f_{1}, f_{2}\right)$ and $g_{i}, h_{j}: \mathbb{R}^{2} \rightarrow \mathbb{R}$ be defined by

$f_{1}\left(x_{1}, x_{2}\right)=\left\{\begin{array}{l}x_{1}+x_{2} \text { if } x_{1} \geq 0, \\ x_{1}^{2}+x_{2} \text { if } x_{1}<0,\end{array} \quad f_{2}\left(x_{1}, x_{2}\right)=x_{2}\right.$,

$g_{1}\left(x_{1}, x_{2}\right)=x_{2}, g_{i}\left(x_{1}, x_{2}\right)=x_{1}^{2} x_{2}-\frac{1}{i}, i \in \mathbb{N} \backslash\{1\}$,

$h_{j}\left(x_{1}, x_{2}\right)=j x_{1}^{3}-x_{1} x_{2}, j \in(0,1)$.

Let $x_{0}=(0,0) . I\left(x_{0}\right)=\{1\}$. By direct computations, one has

$\Omega=\left\{\left(x_{1}, x_{2}\right) \in \mathbb{R}^{2} ? x_{1}=0, x_{2} \leq 0\right\}$,

$T\left(\Omega, x_{0}\right)=\left\{\left(d_{1}, d_{2}\right) \in \mathbb{R}^{2} ? d_{1}=0, d_{2} \leq 0\right\}$,

$\partial_{C} f_{1}\left(x_{0}\right)=\{(y, 1), y \in[0,1]\}, \partial_{C} f_{2}\left(x_{0}\right)=\{(0,1)\}$,

$\left.\partial_{C} g_{1}\left(x_{0}\right)=\{(0,1)\}, \partial_{C} g_{i}\left(x_{0}\right)=(0,0)\right\}, i \in \mathbb{N} \backslash\{1\}$,

$\partial_{C} h_{1}\left(x_{0}\right)=\{(0,0)\}, j \in(0,1)$.

We can check that $f_{1}, f_{2}$ are regular at $x_{0}$ and

$L\left(\Omega, x_{0}\right)=\left\{\left(d_{1}, d_{2}\right) \in \mathbb{R}^{2} d_{1}=0, d_{2} \leq 0\right\} \quad=$

$T\left(\Omega, x_{0}\right)$. Thus the (ACQ) holds. Now we apply

Theorem 3.2. If $x_{0}$ is a local weak efficiency then there are $\alpha_{1}, \alpha_{2} \in \mathbb{R}_{+}^{2} \backslash\{(0,0)\}, \beta_{i}>0$ for $i \in I\left(x_{0}\right)=1$, and $\gamma_{j} \geq 0$ for $j \in J$ such that

$0 \in \sum_{l=1,2}^{m} \alpha_{k} \partial_{k} f_{k}\left(x_{0}\right)+\sum_{i \in I\left(x_{0}\right)} \beta_{i} \partial_{C} g_{i}\left(x_{0}\right)+$

$\sum_{j \in J} \gamma_{j}\left(\partial_{C} h_{j}\left(x_{0}\right) \cup \partial_{C}\left(-h_{j}\right)\left(x_{0}\right)\right)$.

$\alpha_{1}(y, 1)+\alpha_{2}(0,1)+\beta_{1}(0,1)=(0,0)$
Consequently $\alpha_{1}+\alpha_{2}+\beta_{1}=0$, a contradiction. According to Theorem 3.2, $(0,0)$ is not a local weak efficiency of $(\mathrm{P})$.

\section{LIST OF ABBREVIATION}

ACQ: Abadie constraint qualification

KKT: Karush-Kuhn-Tucker

MFCQ: Mangasarian-Fromovitz constraint qualification

PLV: Pshenichnyi-Levin-Valadire

SIP: Semi-infinite multiobjective optimization

\section{CONFLICT OF INTEREST}

We declare that there is no conflict of whatsoever involved in publishing this research.

\section{AUTHOR S' CONTRIBUTIONS}

All authors contributed equally to this work. All authors have read and agreed to the published version of the manuscript.

\section{ACKNOWLEDGMENT}

This research is funded by Vietnam National University HoChiMinh City (VNU-HCM), grant no. C2020-18-03. We are very grateful to the anonymous referees for their valuable remarks and suggestions.

\section{REFERENCES}

1. Gorberna MA, López MA. Linear Semi-infinite Optimization. Wiley, Chichester. 1998;

2. Hettich R, Kortanek O. Semi-infinite programming: Theory, methods, and applications. SIAM Rev. 1993;(35):380-429. Available from: https://doi.org/10.1137/1035089.

3. Reemtsen R, Ruckmann JJ. Semi-Infinite Programming. Nonconvex Optimization and its Applications. Kluwer Academic. Boston . 1998;Available from: https://doi.org/10.1007/978-14757-2868-2.

4. Gorberna MA, López MA, Pastor J. Farkas-Minkowski system in semi-infinite programming. Appl. Math. Optim. 1981;(7):295308. Available from: https://doi.org/10.1007/BF01442122.

5. Puente R, VeraDe VN. Locally farkas minkowski linear inequality systems. TOP. 1999;(7):103-121. Available from: https: //doi.org/10.1007/BF02564714.

6. López MA, Vercher E. Optimality conditions for nondifferentiable convex semi-infinite programming. Math. Program. 1983;(27):307-319. Available from: https://doi.org/10.1007/ BF02591906.

7. Li W, Nahak C, Singer I. Constraint qualifications in semi-infinite systems of convex inequalities. SIAM J. Optim. 2000;(11):31-52. Available from: https://doi.org/10.1137/ S1052623499355247.

8. Stein O. On constraint qualifications in nonsmooth optimization. J. Optim. Theory. Apll. 2004;(121):647-671. Available from: https://doi.org/10.1023/B:JOTA.0000037607.48762.45.

9. Mordukhovich BS, Nghia TTA. Constraint qualifications and optimality conditions for nonconvex semi-infinite and infinite programs. Math. Program. 2013;(139):271-300. Available from: https://doi.org/10.1007/s10107-013-0672-X.

10. Zheng $X Y$, Yang X. Lagrange multipliers in nonsmooth semiinfinite optimization problems. Math. Oper. Res;32:168-181. Available from: https://doi.org/10.1287/moor.1060.0234. 
11. Kanzi N, Nobakhtian S. Optimality conditions for nonsmooth semi-infinite multiobjective programming. Optim. Lett. 2014;(8):1517-1528. Available from: https://doi.org/10. 1007/s11590-013-0683-9.

12. Kanzi N. Necessary optimality conditions for nonsmooth semi-infinite programming problems. J. Global Optim. 2011;(49):713-725. Available from: https://doi.org/10.1007/s10898-010-9561-5.

13. Chuong TD, Kim DS. Nonsmooth semi-infinite multiobjective optimization problems. J. Optim. Theory Appl. 2014;(160):748-762. Available from: https://doi.org/10.1007/ s10957-013-0314-8.

14. Kanzi N. Constraint qualifications in semi-infinite systems and their applications in nonsmooth semi-infinite problems with mixed constraints. SIAM J. Optim. 2014;(24):559-572. Avail- able from: https://doi.org/10.1137/130910002.

15. Clarke FH. Optimization and Nonsmooth Analysis. Wiley, Interscience. 1983:

16. Gfrerer $\mathrm{H}$. On directional metric subregularity and secondorder optimality conditions for a class of nonsmooth mathematical programs. SIAM J. Optim. 2013;(23):632-665. Available from: https://doi.org/10.1137/120891216.

17. Rockafellar RT, Wets JB. Variational Analysis. SpringerVerlag, Berlin. 1998;Available from: https://doi.org/10.1007/ 978-3-642-02431-3.

18. Jiménez B, Novo V. Alternative theorems and necessary optimality conditions for directionally differentiable multiobjective programs. J Convex Anal. 2002;(9):97-116.

19. Rockafellar RT. Convex Analysis. Princeton University Press, Princeton, NJ . 1970. 